\title{
IL Molto Amato Cuore di Ghismonda: RIFLESSIONI SULL'EUCARISTIA E SUL Santo Graal
}

\author{
Fabiano Dalla Bona*
}

ABSTRACT: Il presente paper cerca di tracciare delle possibili convergenze tra la novella di Ghismonda (Decameron IV, 9) con il mito del Santo Graal e con il sacramento dell'Eucaristia, basandosi sugli scritti di Le Goff, Charbonneau-Lassai e Camporesi, tra altri. Nel sacramento cattolico, Cristo offre il suo corpo e il suo sangue agli apostoli affinché, consumandoli, entrino in comunione con Lui nella vita eterna, annunciando la basa dell'escatologia cristiana. Ghismonda, dopo aver bevuto il sangue intriso di veleno, che lei stessa aveva versato sul cuore dell'amante ucciso dal padre, entra in comunione con Guiscardo in una vita eterna, consumando quell'amore interrotto dal padre e indicando la "sua" escatologia. I discepoli bevvero il sangue di Cristo dal Graal: Ghismonda bevve il sangue di Guiscardo da una coppa d'oro. Coincidenza o allusione?

PAROLE CHIAVE: Boccaccio; Decameron; Ghismonda; Eucaristia; Santo Graal.

RESUMO: O presente artigo procura traçar possíveis convergências da novela de Ghismonda (Decameron IV, 9) com o mito do Santo Graal e o sacramento da Eucaristia, baseando-se nos escritos de Le Goff, Charbonneau-Lassy e Camporesi, entre outros. No sacramento católico, Cristo oferece seu corpo e seu sangue aos apóstolos a fim de que, consumindo-os, entrem em comunhão com Ele na vida

\footnotetext{
* Faculdade de Letras - UFRJ
} 
eterna, anunciando a base da escatologia cristã. Ghismonda, após ter bebido o sangue misturado ao veneno, que ela mesma derramara sobre o coração do amante assassinado pelo pai, entra em comunhão com Guiscardo, também para uma vida eterna, consumando aquele amor que fora interrompido pelo pai e indicando a "sua" escatologia. Os discípulos beberam o sangue de Cristo do Graal: Ghismonda bebeu o sangue de Guiscardo de um cálice de ouro. Coincidência ou alusão?

PALAVRAS-CHAVE: Boccaccio; Decameron; Ghismonda; Eucaristia; Santo Graal

ABSTRACT: This paper tries to trace possible convergences between the tale of Ghismonda (Decameron IV, 9) and the myth of the Holy Grail and the sacrament of the Eucharist, based on the writings of Le Goff, Charbonneau-Lassy and Camporesi, among others. In the Catholic sacrament, Christ offers his body and blood to the apostles so that by consuming them, they enter into communion with Him in eternal life, announcing the foundation of Christian eschatology. After drinking the blood mixed with poison, she poured over the lover's organ murdered by her father, Ghismonda enters into communion with Guiscard, also for eternal life, concluding that love which her father interrupted and stating "her" eschatology. The disciples drank Christ's blood from the Grail: Ghismonda drank Guiscardo's blood from a golden cup. Coincidence or allusion?

KEY WORDS: Boccaccio; Decameron; Ghismonda; Eucharist; Holy Grail. 


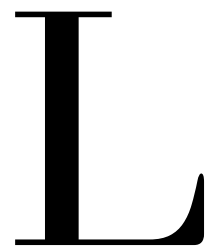

a novella di Tancredi e Ghismonda, onde "Tancredi prenze di Salerno uccide l'amante della figliuola e mandale il cuore in una coppa d'oro; la quale, messa sopr'esso acqua avvelenata, quella si bee, e così muore" (BOCCACCIO, 2002, p. 337), conclude il trittico di novelle tragiche della IV Giornata del Decameron, insieme a Lisabetta da Messina e alla moglie di Guglielmo di Rossiglione, in cui le donne che ebbero una fine tragica sono le protagoniste.

L'analisi di Surdich ci rivela:

Non è un caso che la triade di novelle che ha a protagoniste le menzionate figure femminili (la 1, la 5, la 9) si ponga un gradino più alto rispetto alle altre, sia per l'assolutezza del tragico da esse esibito, che non reclama alcun preambolo 
introduttivo, sia perché tutt'e tre le novelle fanno un uso quanto mai evidente di un apparato simbolico di alta capacità evocativa. (SURDICH, 2002, p. 146).

Denis corrobora le parole di Surdich quando afferma, a proposito delle stesse novelle:

Tutte queste immagini s'iscrivono nella corrente di pensiero cortese che valorizza il concetto dell'unione dei cuori, il loro dono reciproco, e fanno dell'amore un tesoro destinato ad essere preservato, così come è stato l'eroismo fedele del suo equivalente epico. (DENIS, 1998, p. 103).

La stessa opinione viene condivisa da Lupton:

In this triad of tales, the symbolizing stroke of dismemberment gives way to an act of cannibalism incorporation, the nonsymbolizing ingestion of a traumatic alterity. Thus Ghismonda receives the dislodged heart of her lover in a golden chalice that cradles its (dis)contents like a reliquary or a communion cup; the quasi cannibalism of Ghismonda's deathly drink is radicalized in the scandal of 4-9's dainty meal served in a silver tureen, and the chalice enclosing Guiscardo's heart mutates into a pot of basil in Lisabetta's tale (4-5). (LUPTON, 1998, p. 12)

Nella quarta giornata si registra una costante presenza del "cuore" sin dalla prima novella, in cui Ghismonda segna la storia del topos dell'ostensione dei cuori sanguinanti, bevendo il veleno che versa nella coppa con il cuore dell' amato che il padre, il "prenze", principe Tancredi, le ha fatto pervenire, nel suo letto, diventato insieme tomba e altare.

Nella visione dello storico Le Goff, il cuore

è definito da Aristotele come l'origine della sensazione, e l'aristotelismo medievale recupera il tema. Sant'Agostino fa del cuore la sede dell' "uomo interiore". Nel XII secolo, il secolo della grande affermazione dell'amore, si impongono parallelamente l'amore sacro, esaltato dai tanti commentari al Cantico del cantici, e l'amore profano, che assume le forme dell'amore cortese. (LE GOFF, 2011, p. 96). 
Il cuore è stato a lungo utilizzato come un simbolo riferito all'emotività, moralità e spiritualità insite nell'essere umano. Dato che un tempo si riteneva il cuore la sede della mente umana, la parola cuore continua ad essere utilizzata poeticamente per fare riferimento all'anima, e le rappresentazioni stilizzate di cuori sono molto diffuse in simboli che rappresentano l'amore. Tradizionalmente, nel folklore, il cuore viene disegnato in forma stilizzata, in genere di color rosso, per via della valenza che questo colore possiede nell'indicare sia il sangue sia, altrettanto frequentemente, la passione.

Sull'immagine del cuore come epicentro della passione, scrive Franco:

L'unione più intima che possa aversi è quella che passa tra i cuori: perocchè in questa sono le stesse affezioni, lo stesso volere e non volere, lo stesso amore, lo stesso odio, la trasformazione quasi direi della volontà dell'uno in quella dell'altro. Questa unione è la più quiete, perché tra gli esseri ragionevoli non nasce inquietudine se non dalla diversità del volere: è la più dolce perché è quella che risiede nella potenza più capace di gaudio qual è la volontà: è la sola veramente affettuosa, perché si consuma nel centro di tutti gli affetti: è quella che sublima e deprime l'uomo, perché l'uomo è quello che è il suo amore. (FRANCO, 1868, p. 208).

Dall'era cristiana il cuore è stato considerato anche l'emblema dell'amor sacro. Infatti il simbolismo del cuore prende un'altra dimensione attraverso il culto del Sacro Cuore di Gesù e la leggenda del Santo Graal.

Il termine graal viene dallo scrittore francese Chrétien de Troyes (c. 1135 - c. 1191) autore del poema intitolato Le Roman de Perceval ou le conte du Graal, il cui committente fu Filippo I d'Alsazia (1143-1191). Chrétien de Troyes morì nel 1190-91 prima di completare l'opera. Nel poema, un giovane cavaliere visita il castello del Re Pescatore dove vede una strana processione nella quale è presente un piatto d'oro incastonato di gemme e chiamato "graal". Esso era un oggetto sacro utilizzato per trasportare l'ostia consacrata. Nella processione, vede anche un ragazzo che trasportava una lancia sanguinante dalla punta, probabilmente un riferimento alla lancia che ferì il costato di Gesù sulla croce (Giovanni 19,34) e perciò si finì col collegare questo graal con un artefatto della Passione. Poiché la storia non fu mai finita, è impossibile sapere cosa intendesse l'autore (BAIGHEN, 1999, p. 306). 
Cionondimeno, questa storia incompleta divenne la fonte da cui attinsero numerosi autori che si riproposero di completarla. Le due opere più famose così ottenute sono il Parzifal di Wolfram von Eschenbach e La Storia del Graal di Robert de Boron. Boron, che operò agli inizi del XIII secolo, fu il primo autore a suggerire che il graal potesse essere identificato con un artefatto usato nell'Ultima Cena. Egli scrisse che la coppa fu trasportata da Gerusalemme in Occidente (forse in Gran Bretagna), dove divenne il fulcro della leggenda di Re Artù.

La maggior parte degli scrittori medievali descrive il graal come una specie di coppa o piatto, in riferimento sia al piatto in cui Giuda intinse le proprie dita, sia alla coppa da cui i discepoli bevvero durante l'Ultima Cena (Matteo 26, 23-27).

La parola graal potrebbe derivare dal latino gradalis che si riferisce a una larga base da portata per servire le carni. Potrebbe anche essere legata al greco krater, un imponente calice con maniglie. Quest'ultima è l'interpretazione che gli scrittori successivi preferirono. Ma gli scrittori medievali spesso descrivono un forte legame tra il graal e il corpo e il sangue di Cristo, dimostrando come fosse, anch'esso, un simbolo dell'Eucaristia.

Già nel XII secolo, la chiesa cattolica dovette affrontare, in Francia, la minaccia degli eretici catari, che sfidavano l'insegnamento della Chiesa secondo il quale l'Eucaristia era la via per la salvezza. Fu allora coniato il termine transustanziazione per descrivere la trasformazione del pane e del vino, benedetti dal sacerdote, nel corpo e nel sangue di Cristo. Nel 1264, il Papa Urbano IV introdusse in tutta la Chiesa occidentale la festa del Corpus Christi con l'intento di dare una dimostrazione pubblica della presenza di Cristo nell'ostia, ossia di rendere visibile Cristo come l'ostia portata in processione in un ostensorio e di impartire la benedizione eucaristica.

Negli scritti di Ghénon, specie in quello sull'esoterismo cristiano, l'autore francese osserva:

Questa coppa si sostituisce dunque, in un certo senso, al Cuore di Cristo, come ricettacolo del suo sangue; essa, per così dire, ne prende il posto e diviene un simbolo equivalente; ed allora accanto a tutto questo, non è ancora più notevole che già anticamente il vaso fosse un emblema del cuore? D'altronde, la coppa, sotto una forma o l'altra, giuoca, al pari del cuore stesso, un ruolo molto importante in tante antiche tradizioni, e senza dubbio fu così anche presso i celti, poiché è da essi che deriva ciò che costituisce la base stessa o, quantomeno, la trama della 
leggenda del Graal. (GHÉNON, 1997, p. 34).

Sarebbe, dunque, impreciso pensare alla coppa contenente il cuore di Guiscardo come un'interpretazione del mitico graal? Il Boccaccio così narra l'invio del cuore di Guiscardo a Ghismonda da parte di Tancredi dentro la coppa d'oro:

Laonde, venuto il dì seguente, fattasi il prenze venire una grande e bella coppa d'oro e messo in quella il cuor di Guiscardo, per un suo segretissimo famigliare il mandò alla figliuola e imposegli che, quando gliele desse, dicesse: "Il tuo padre ti manda questo, per consolarti di quella cosa che tu più ami, come tu hai lui consolato di ciò che egli più amava." (BOCCACCIO, 2002, p. 345).

E la scena continua:

Alla quale venuto il famigliare e col presento e con le parole del prenze, con forte viso la coppa prese; e quella scoperchiata, come il cuor vide e le parole intese, così ebbe certissimo quello essere il cuor di Guiscardo; per che, levato il viso verso il famigliar, disse: "Non si convenia sepoltura men degna che d'oro a così fatto cuore chente questo è: discretamente in ciò ha il mio padre addoperato." (BOCCACCIO, 2002, p. 345-346).

Quella cosa che tu più ami, nelle parole di Boccaccio, è la cosa che più amava il Cristo e che condivise con i discepoli. Cristo, nella memoria eucaristica, consegna agli apostoli il suo corpo e il suo sangue affinché il sacrificio della croce, ossia la sua immolazione, da lui passi ai discepoli per essere assunto e condiviso. I cristiani, con tale assunzione e condivisione, diventano anch'essi "immagine" del figlio di Dio risorto dalla morte, essendo riconosciuti e identificati dal Padre, secondo la tradizione. Prendere parte al convito eucaristico del corpo e del sangue di Cristo significa diventare "consorti" della sua morte e quindi della sua resurrezione. In coloro che mangiano la carne e bevono il sangue del Signore (Gv 6, 53-77) si avvera l'eterna destinazione pasquale.

Cristo pronuncia le parole che trasformano pane e vino nel suo corpo e nel suo sangue. Accade la transustanziazione. La nostra eroina pronuncia delle parole che danno un senso 
ritualistico al consumo di quella bevanda, mista di veleno e sangue dell'amante morto. Pensiamo anche ad una transustanziazione laica, poiché l'assimilazione di quella bevanda condurrà Ghismonda alla “vita eterna" insieme all'amante già partito. Hic est enim Calix Sanguinis mei, novi et aeterni testamenti (Mt 26,28)

La qual, poi che quanto le parve ebbe pianto, alzato il capo e rasciuttosi gli occhi, disse: "O molto amato cuore, ogni mio uficio verso te è fornito; né più altro mi resta a fare se non di venire con la mia anima a fare alla tua compagnia." E questo detto, si fe' dare l'orcioletto nel quale era l'acqua che il dì avanti aveva fatta, la qual mise nella coppa ove il cuore era da molte delle sue lagrime lavato, e senza alcuna paura postavi la bocca, tutta la bevve, e bevutala, con la coppa in mano se ne salì sopra il suo letto, e quanto più onestamente seppe compose il corpo suo sopra quello, e al suo cuore accostò quello del morto amante, e senza dire alcuna cosa aspettava la morte. (BOCCACCIO, 2002, p. 347).

Ghismonda è commossa tanto quanto Sant'Ambrogio quando esclama "che meraviglia il tuo calice che dà l'ebbrezza. Bevi Cristo, per bere il sangue da cui sei stato redento; bevi Cristo, per bere il Suo discorso" (AMBROGIO, 1980, p. 78). Il linguaggio accomuna, nell'immagine della bevanda e dell'ebbrezza, la realtà eucaristica che permette l'incontro transumanante con il divino.

L'Eucaristia, che provoca l'ebbrezza in Ambrogio, secondo Camporesi, nonostante sia misteriosa e confusa, può essere paragonata a qualcosa di stupefacente, "sentita come una droga dolce e tremenda, come un frammento di carne sacra che estraniava dalla realtà, conduceva altrove, apriva dimensione ignote ai sensi, dilatava la coscienza e la modificava (seppur temporaneamente)" (CAMPORESI, 1987, p. 249). Usa ancora le espressioni "nepente narcotizzante, celeste tranquillante, oppio trascendente" (CAMPORESI, 1987, p. 248). O ancora "prodigiosa medicina dell'anima e del corpo, beatificante viatico notturno, per viaggi dentro spazi inimmaginabili alla luce del giorno, elettuario psicopompo, accompagnatore del fedele in dimensioni nuove, in contrade indescrivibili" (CAMPORESI, 1987, p. 249).

Ecco il mysterium fidei della novella boccacciana. Ghismonda venera il cuore dell'amante morto e lo consuma come se fosse un sacramento. Tramite le sue parole la donna sacralizza l'oggetto e spiega le ragioni per le quali si offre in sacrificio.

Charbonneau-Lassay, in un importante studio sul simbolismo del cuore di Cristo, sostiene: 
Cuore e parola non sono che prolungamenti della stessa cosa; la voce è la rissonanza del cuore; sono come la sorgente e il flusso, come la ferita e il sangue dalla quale sgorga, come la rosa e il suo profumo, come l'albero e i suoi frutti, come il braciere e il calore che emana. "La bocca parla dalla pienezza del cuore", ha detto Gesù. (CHARBONNEAU-LASSAY, 1995, p. 26).

Comunemente, quando parliamo del simbolo, intendiamo un procedimento conoscitivo semplice ed immediato, che si avvale di un segno sensibile o di un'immagine che fa da supporto ad un concetto da trasmettere. Ricoeur definisce simbolo come "ogni struttura di significato dove un senso diretto, primario, letterale, designa per sovrabbondanza un altro senso indiretto, secondario, figurato, che non può essere appreso se non attraverso il primo" (RICOEUR, 2007, p. 26).

E le parole di Ghismonda sono tanto simboliche in questo senso:

Questo detto, rivolta sopra la coppa la quale stretta teneva, il cuor riguardando disse: "Ahi! dolcissimo albergo di tutti i miei piaceri, mala detta sia la crudeltà di colui che con gli occhi della fronte or mi ti fa vedere! Assai m'era con quegli della mente riguardarti a ciascuna ora. Tu hai il tuo corso fornito, e di tale chente la fortuna tel concedette ti se' spacciato; venuto se' alla fine alla qual ciascun corre; lasciate hai le miserie del mondo e le fatiche, e dal tuo nemico medesimo quella sepoltura hai che il tuo valore ha meritata. Niuna cosa ti mancava ad aver compiute esequie, se non le lagrime di colei la qual tu vivendo cotanto amasti; le quali acciò che tu l'avessi, pose Iddio nel l'animo al mio dispietato padre che a me ti mandasse, e io le ti darò, come che di morire con gli occhi asciutti e con viso da niuna cosa spaventato proposto avessi; e dateleti, senza alcuno indugio farò che la mia anima si congiugnerà con quella, adoperandol tu, che tu già cotanto cara guardasti. E con qual compagnia ne potre' io andar più contenta o meglio sicura ai luoghi non conosciuti che con lei? Io son certa che ella è ancora quincentro e riguarda i luoghi de' suoi diletti e de' miei; e come colei che ancor son certa che m'ama, aspetta la mia, dalla quale sommamente è amata." (BOCCACCIO, 2002, p. 346). 
Ghismonda usa la parola e, contrariamente alla piagnucolosa Lisabetta da Messina, seppur anch'ella pianga, dimostra grande cortesia. In un recente articolo giornalistico sulle commemorazioni boccacciane, Barilli scrive che Ghismonda

pronuncia un'orazione sublime, che si solleva al livello della tragedia e potrebbe essere declamata sulle scene. O meglio, è l'avvocato Boccaccio a metterle in bocca una delle più belle e commoventi orazioni di tutti i tempi. Questo in deroga, evidentemente, ai canoni di una piatta verosimiglianza psichica, in primo luogo per la ragione a lui ben nota che le donne in quegli anni non erano capaci di tanta eloquenza, e poi per l'altra ragione ancor più cogente che chi è in uno stato di profonda emozione non riesce quasi a parlare. (BARILLI, 2013, p. 19).

Un importante studio di Cosentino sulle eroine tragiche, nonostante tratti delle tragedie fiorentine del Cinquecento e metta in evidenza, tra le altre, le figure di Didone, Rosmunda, Ifigenia, Semiramide e Tullia, ci fornisce un profilo abbastanza aderente all'atteggiamento di Ghismonda. Diremmo, addirittura, che la Cosentino abbia dimenticato due altre grandi eroine quali Artemisia e Sofonisba che, meglio ancora delle altre, possono essere accostate all'eroina tragica del Boccaccio. Ambedue presero del veleno per suicidarsi, così come fece la nostra Ghismonda. Ma ecco come la Cosentino definisce il carattere eroico delle donne:

Ogni personaggio femminile risulta essere il frutto di un sapiente lavoro di intarsio, che fonde antico e moderno, leggende derivate dalla storia recente e miti del più lontano passato: se ne ricavano diverse tipologie di donna che tuttavia hanno in comune alcuni caratteri fondamentali. L'eccezionale energia di cui sono dotate è la testimonianza di un coraggio fuori dalla norma e, per questo, di per sé sospetto e pericoloso. [...] La virtù femminile, ogni qual volta di domestica cerca di farsi politica e civile, rischia di trasformarsi in acciecamento, di minacciare la severa razionalità maschile con un eccesso di passionalità. (COSENTINO, 2006, p. 7273).

E aggiunge: 
L'ammirazione per la forza d'animo femminile porta con sé stupore, malessere, paura; anzi il confine fra bene e male rimane, in definitiva, difficile da tracciare con chiarezza. Non a caso, le protagoniste femminili di queste tragedie sono donne che utilizzano la trasgressione per affermare la propria individualità: esse disobbediscono, ingannano, tradiscono, giungono persino ad uccidere. Alla base della raffigurazione convenzionale dell'eroina sta la necessità di introdurre elementi che non sono del tutto conformi alla rappresentazione tradizionale della donna. (COSENTINO, 2006, p. 73).

Invece Picone è dell'opinione che la novella trova

materia ben identificata negli archivi della cultura classica e romanza: quella relativa all'amore-passione che, combinato con l'avversa fortuna, porta gli amanti alla morte; quella che partendo dalle Heroides ovidiane, arriva fino a Paolo e Francesca, passando attraverso Tristano e Isotta. (PICONE, 2004, p. 120).

Anche per Tortelli, grazie all'atteggiamento altero, Ghismonda appartiene alla schiera delle eroine tragiche:

Ghismunda si pone subito come una eroina tragica: lo statuario comportamento e le razionali argomentazioni, sostenute a difesa della sua scelta, degne di una vera regina dell'ars loquendi et dictandi la ritraggono come un personaggio di nobile e alto sentire. Le lacrime per lei sono segno di debolezza e di umiliazione, per questo propende per un dolore altero e decoroso da contrapporre all'insensata autorità paterna. Anche quando le viene consegnata la coppa con il cuore dell'amato, la donna, che non ha rinunciato al proposito di infliggersi la morte, si china sul cuore per piangere, ma questo gesto è condotto con nobile discrezione e riservatezza, senza abbandono irrazionale all'emozione, quindi senza tradire il suo temperamento fiero. (TORTELLI, 2013, on-line).

La Ghismonda del Boccaccio è una di queste donne che disobbediscono, che trasgrediscono le consuetudine del loro tempo. Ghismonda s'innamora di un uomo di classe sociale inferiore, atto inconcepibile nella società mercantile fiorentina del Trecento. E ancora, sulla trasgressione: 
L'eccezionale energia di cui si fanno portatrici è la testimonianza di un coraggio fuori dal comune, che da virtù rischia talvolta di trasformarsi in vizio. Labile è infatti il confine fra bene e male, dal momento che proprio queste tragedie costituiscono il luogo per l'affermazione di una trasgressione di segno femminile. Il dramma diventa infatti il campo di investigazione per la definizione di un modello di donna protagonista dell'azione, la cui condizione ambigua, insieme di "vittima" e di "colpevole", consente l'affermazione di uma complessità psicologica nuova. L'eroe o, come in questi casi, l'eroina oscilla perennemente tra il tipo, ovvero un caso illustre, che invita a riflettere sulla precarietà dell'esistenza umana o sull'importanza della virtù, e il modello, inteso come paradigma a cui conformare le proprie azioni. (COSENTINO, 2006, p. 73).

Discorrendo sulle trasgressioni e sulle somiglianze e differenze tra la Francesca da Rimini di Dante e la Ghismonda di Boccaccio, Sanguineti, basandosi sulle affermazioni di Auerbach, scrive:

La persona che ha provato l'orgasmo è soddisfatta. Non è depressa, irriquieta, irritabile, malinconica o polemica. Lontana dal cliché misogino della fragilità femminile, padrona di sé, sessualmente appagata. Ghismonda non ha spirito di rivalsa, non desidera vendetta, non teme la morte. (SANGUINETI, 2004, p. 199).

Già Valesio sostiene che sia impossibile pensare che Ghismonda sia venuta al mondo senza riconoscere in Francesca da Rimini una sua cruciale genealogia; il discorso di Ghismonda si sovrappone al silenzio di Guiscardo così come quello di Francesca integra il silenzio di Paolo: "Mentre che l'uno spirto questo disse,/ l'altro piangea, sì che di pietade io venni men com'io morisse" (VALESIO, 1998, p. 79).

Mentre Francesca era turbata dall'idea del peccato, Ghismonda non lo è. Il suo discorso si richiama al potere del desiderio carnale e il cuore assume delle valenze erotiche. Il sanguinario episodio dell'offerta del cuore dentro la coppa d'argento è una scena ancora più erotica di quella dell'atto sessuale compiuto dagli amanti.

Per Sanguineti la differenza tra l'eroina dantesca e quella boccacciana è la seguente:

A differenza dei peccatori carnali condannati nell'inferno, sofferenti di una 
scissione della personalità, che li fa vergognosi della loro stessa umanità degradata, invocanti infatti pietà del proprio male perverso, l'eroina di Boccaccio, agendo secondo natura e coscienza, lungi dal sottomettere la ragione al talento, oppone, alla teologia del verbo fatto carne, un'antropologia autosufficiente, dove la radice dell'essere umano è l'essere umano, presentandosi come "figliuola di carne e non di pietra o di ferro" e "generata di carne". (SANGUINETI, 2007, p. 9).

Per Doueihi, invece, Ghismonda invoca degli ideali cristiani come veicolo per la sua critica all'ordine sociale del suo tempo. Il suo amore, letteralmente, la libera dalla sua condizione familiare e sociale; ella assicura la sua indipendenza e autonomia in nome delle leggi della natura contro le accuse e la crudeltà del padre (DOUEIHI, 1998, p. 53).

Partecipare al sangue di Cristo nell'eucaristia significa entrare nell'alleanza nuova, escatologica, inaugurata dalla croce di Cristo, in cui sono comunicati ai credenti il perdono e la remissione dei peccati per una piena comunione di vita con Dio, così come Ghismonda che beve il liquido versato sul cuore entra in comunione con il sangue dell'amante morto e preannuncia la sua escatologia. "L'angoscia del pianto non lasciò rispondere al prenze. Laonde la giovane, al suo fine esser venuta sentendosi strignendosi al petto il morto cuore, disse: 'Rimanete con Dio, ché io mi parto."” (BOCCACCIO, 2002, p. 348).

Strappini invece è dell'opinione che

Riservandosi la morte come scelta autonoma e consapevole, ecco la più piena affermazione di sé e della propria individualità, secondo quella prospettiva di carattere stoico, propria anche di altri personaggi del Decameron, che contemplano il suicidio come atto di libertà. (STRAPPINI, 2010, p. 24).

Nel Decameron, Ghismonda è collocata in quella giornata dedicata agli amanti che si sono abbandonati nella pratica terrena dell'eros, alla incontenibile forza della loro pulsione erotica che, combinata con l'avversa fortuna, li ha condotti alla morte, secondo il binomio eros/thanatos. La tomba comune, per Boccaccio, è, tuttavia, il mezzo attraverso il quale gli opposti vita/morte si conciliano grazie all'amore. Per l'autore la morte, lungi dall'essere la dimensione sublime della vita, il prezzo ultimo da pagare per ottenere l'amore, è la negazione della vita a cui solo la memoria degli uomini può riparare. 


\section{Riferimenti bibliografici}

AMBROGIO, Santo. Commento a dodici Salmi. Trad. L. F. Pizzolato. Roma: SAEMO 7, 1980.

BAIGHEN, Michael et al. Il Santo Graal: una catena lunga duemila anni. 17. ed. Trad. Roberta Rambelli. Milano: Mondadori, 1999.

BOCCACCIO, Giovanni. Decameron. Milano: Mondadori, 2002.

CHARBONNEAU-LASSAY, Louis. Il giardino del Cristo ferito: il vulnerario e il florario di Cristo. Trad. S. Palamidessi. Roma: Edizioni Arkeios, 1995.

COSENTINO, Paola. Tragiche eroine: virtù femminile fra poesia e trattati sul comportamento. Italique on line, IX, p. 68-99, 2006. Indirizzo on-line: http://index.108.html . Ultimo accesso 14 nov. 2013.

DENIS, François. Coeur arraché / coeur mangé: modulations. Études littéraires, v. 31, n. 1, p. 95-108, 1998. Indirizzo on-line: http://erudit.org. Ultimo accesso 18 nov.2013.

DOUEIHI, Milad. A perverse history of the human heart. Cambridge-London: Harvard University Press, 1998.

FRANCO, Secondo. Dalla devozione al Sacro Cuore SS. di Gesù e delle sue eccellenze. 8. ed. Roma: Tipografia della S. C. de Propaganda Fide, 1868.

GHÉNON, René. L'esoterismo cristiano: San Bernardo. Trad. Calogero Cammarata. Carmagnola: Oggero Editore, 1997.

LE GOFF, Jacques. L'immaginario medievale. Trad. Vivanti Salmon. 9a ed. Roma-Bari: Laterza, 2011.

LUPTON, Julia R. Secularization and its symptoms: Boccaccio's Decameron. In: MURRAY, T; SMITH, A. K. Repossessions: psychoanalysis and the phantasms of early modern culture. Minneapolis: University of Minnesota Press, 1998. p. 3-22.

PICONE, Michelangelo. L"“amoroso sangue": la quarta giornata. In: Introduzione al Decameron. Firenze: M. Mersica, 2004.

RICOEUR, Paul. Il conflitto delle interpretazioni. Trad. Rodolfo Balzarotti. 4. ed. Milano: Jaca Books, 2007.

SANGUINETI, Federico G. Quarta giornata. Heliotropia, no 4-1.2, 2007, p. 1-19. Indirizzo on-line: $\underline{\text { http:// }}$ www.heliotropia.org/04-0102/sanguineti.pdf. Ultimo accesso 15 dic. 2013

. Francesca e Ghismonda. Tenzone, Madrid, n. 5, p. 187-21, 2004. Indirizzo on-line: http:// pendientedemigracion.ucm.es/info/italiano/acd/tenzone /t5/Federico-Garabello-Sanguineti-Tenzone-5.pdf. Ultimo accesso 13 dic. 2013.

STRAPPINI, Lucia. Tragico e comico in due novelle del Decameron. Revista di Italianística. São Paulo: EDUSP, XIX-XX, p. 16-29.

SURDICH, Luigi. Boccaccio. Roma-Bari: Laterza, 2002.

TORTELLI, Elisabetta. Emozioni estreme: il riso e il pianto nel Decameron. Griselda online: portale di letteratura. Indirizzo on-line: http://www.griseldaonline.it/didattica/ riso-pianto-decameron-tortelli.html. Pubblicato il 15 gen. 2013. Ultimo accesso 14 febb. 2014.

VALESIO, Paolo. The fierce dove. In: MANDELBAUM, A.; OLDCORN, A.; ROSS, C. (Org.). Lectura Dantis: Inferno. Berkeley-Los Angeles: University of California Press, 1998. p. 63-83. 